\title{
A Experiência dos Profissionais de Saúde no Uso de um Ambiente de Inovação Educacional
}

\author{
Amadeu S. Campos Filho', Matheus S. Monteiro ${ }^{1,2}$ \\ ${ }^{1}$ Núcleo de Telessaúde (NUTES) - Universidade Federal de Pernambuco (UFPE) \\ Recife - PE - Brasil \\ ${ }^{2}$ Centro de Informática - Universidade Federal de Pernambuco (CIn - UFPE) \\ Recife - PE - Brasil \\ \{amadeu.campos, matheus.monteiro\}@nutes.ufpe.br
}

\begin{abstract}
Education is a complex process and the incorporation of technology in health education continues to be a growing trend worldwide. Moreover, the constant changes in health care result in the need for new skills and training for health professionals through a fast and effective teaching-learning process. In this context, the Pernambuco Telehealth Center developed a technological innovation platform (INDU). This study aims to analyze the usability of INDU. The methodology used was a case study with the final participation of 103 students. The evaluation was done by a usability questionnaire to students. The study results showed that INDU had a good rating in terms of usability and interaction it was simple and agreeable.
\end{abstract}

Resumo. A educação é um processo complexo e a incorporação de tecnologia em educação para a saúde continua a ser uma tendência crescente em todo o mundo. Além disso, as constantes mudanças nos cuidados a saúde resultam na necessidade de novas capacitações e treinamentos para os profissionais de saúde através de um processo de ensino-aprendizagem rápido e eficaz. Nesse contexto, o Núcleo de Telessaúde de Pernambuco desenvolveu uma plataforma de Inovação Educacional (INDU). Este estudo tem como objetivo analisar a usabilidade do INDU. A metodologia utilizada foi um estudo de caso com a participação final de 103 alunos. Uma avaliação foi feita através de um questionário de usabilidade aplicado aos alunos. Os resultados do estudo mostraram que o INDU teve uma classificação boa em termos de usabilidade e a interação nele foi simples e agradável.

\section{Introdução}

A educação articula os processos dinâmicos de ensino e aprendizagem, compreensão e construção contínua do conhecimento. É um fato observado em qualquer sociedade e é responsável pela sua manutenção e propagação do conhecimento para as gerações que seguem as formas culturais de ser, de viver e de agir necessário para a comunicação e ajustamento de um membro do grupo ou sociedade. Na educação, o comportamento flexível é exigido tanto dos professores como uma habilidade a ser adquirida, como aos alunos, futuros trabalhadores. Por outro lado, a educação em saúde é um amplo conjunto de conceitos e estratégias, o que antes era o meio de promoção da saúde e tratamento da 
V Congresso Brasileiro de Informática na Educação (CBIE 2016)

Anais do XXVII Simpósio Brasileiro de Informática na Educação (SBIE 2016)

doença, mas hoje sabemos que é um conjunto de ações que ajudam o indivíduo não só prevenir a doença, mas ajuda a fazer tem um estilo de vida saudável.

Ao relacionar essa concepção de educação com a profissão de saúde, considerada também como prática social compreende-se que, em todas as ações de saúde, estão inseridas ações educativas. Além disso, a educação em saúde foi reestruturada para ampliar a sua margem de ofertas nos serviços de saúde. Pode-se caracterizar a educação em saúde com uma disciplina que fornece um campo de prática que começa ao nível das relações sociais, geralmente são estruturados por profissionais de saúde, entre si, com as instituições e os seus utilizadores no desenvolvimento de suas atividades diárias.

Além disso, a dimensão e contexto vivenciado com o desenvolvimento da sociedade da informação e a generalizada difusão das tecnologias da informação e da comunicação (TIC) permitiram novos cenários para o processo de ensino-aprendizagem nas duas modalidades de ensino: presencial e a distância. As TIC, especialmente a internet, são poderosos recursos para a comunicação e facilitam o acesso às informações especialmente sobre saúde [Novaes et al. 2012], [WHO 2016]. As Instituições de uma forma geral incorporaram as TIC na sua prática pedagógica, na gestão e na administração de programas educacionais em suas extensões. As TIC também possibilitaram que a construção dessa atualização e que o treinamento fosse realizado através do ensino a distância (EAD) ou tele-educação, estratégia esta que conquista maior adesão de educandos e profissionais de saúde que buscam se atualizar, capacitar e sensibilizar através do conhecimento adquirido às comunidades localizadas distantes dos grandes centros urbanos [Knowles 2008], [Zollo et al. 1999], [Curran, Fleet e Kirby 2006]. Sendo assim, a tele-educação mostra-se como uma possibilidade promissora [Graeff-Martins 2008], [WHO 2016].

Por outro lado, alguns aspectos dificultam a ampliação da utilização do serviço de Tele-educação para profissionais de saúde no Brasil estão relacionados à baixa qualidade ou ausência de conexão à internet, a falta de informatização nas Unidades de Saúde da Família, a alta rotatividade dos profissionais da atenção primária e da gestão, a dificuldade em tornar a tele-educação uma rotina do dia-a-dia das unidades e, principalmente, a falta de conhecimento tecnológico da equipe de trabalho quanto aos processos e tecnologias envolvidas na execução das práticas educativas, resultando assim no não reconhecimento do serviço como prática associada e complementar às demais atividades da unidade. Esse tipo de proposta não apresenta grandes custos e proporciona facilidade no processo ensino-aprendizagem, pois facilita para $o$ profissional que não possui tempo disponível poder acessar no seu horário livre.

Neste contexto, surgiu além da necessidade do desenvolvimento de um ambiente de Inovação Educacional (INDU ${ }^{1}$ ) com a proposta de ser um recurso educacional inovador para promover a educação e disseminar o conhecimento nas áreas de saúde e tecnologias associadas, a avaliação de usabilidade da sua interface. O INDU é um Ambiente Virtual de Aprendizagem (AVA) baseado no MOODLE e foi desenvolvido pelo Núcleo de Telessaúde - NUTES dentro do Programa Nacional Telessaúde Brasil Redes em Pernambuco (RedeNUTES) que é uma ação do Ministério da Saúde do

\footnotetext{
${ }^{1}$ www.nutes.ufpe.br/indu
} 
V Congresso Brasileiro de Informática na Educação (CBIE 2016)

Anais do XXVII Simpósio Brasileiro de Informática na Educação (SBIE 2016)

Brasil $^{2}$. Tendo em vista as possibilidades do campo conceitual e prático dos AVA na Educação a distância (EAD) em meios as ferramentas disponíveis para aprendizagem em cursos on-line, foi possível um mapeamento estrutural dos cursos ofertados na modalidade a distância da RedeNUTES. O INDU oferece cursos e bibliotecas digitais com conteúdos nas mais diversas áreas de conhecimento da saúde e tecnologias da informação em saúde.

Assim sendo, este estudo procurou entender o perfil tecnológico dos alunos que participaram do experimento, assim como as dificuldades que eles tiveram na utilização do INDU. O estudo explorou questões de usabilidade sobre os ambientes virtuais de aprendizagem. O objetivo do artigo desenvolvido foi descrever a avaliação da usabilidade do INDU no processo de ensino-aprendizagem dos profissionais de saúde.

\section{Metodologia}

A metodologia usada para a análise da usabilidade do INDU foi adaptada de Campos Filho, Novaes e Gomes (2015) onde os autores utilizaram técnicas e instrumentos para a aferição da usabilidade centrada no usuário. A adaptação realizada a metodologia de Campos Filho, Novaes e Gomes (2015) foi em relação ao metodo de coleta das informações e a avaliação de usabilidade onde escolhemos utilizar outro instrumento de usabilidade chamado System Usability Scale (SUS). Assim, o estudo foi realizado em três fases conforme a figura 1. A primeira fase foi composta da apresentação do estudo (Justificativa, Relevância, objetivo e metodologia) para os alunos e o preenchimento de um questionário demográfico para coletar o perfil tecnológico da amostra. A segunda fase foi a realização da disciplina durante o período letivo. A terceira fase foi a aplicação do questionário de Usabilidade e satisfação.

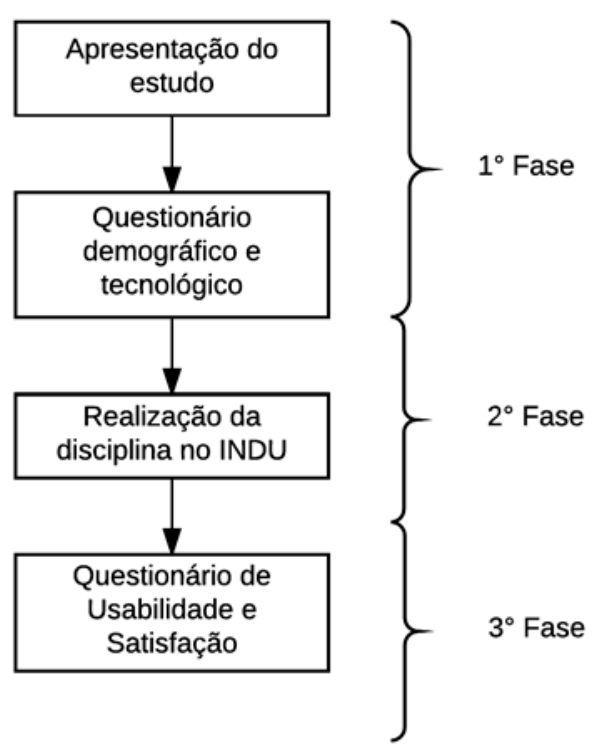

Figura 1 - Metodologia do estudo

\footnotetext{
${ }^{2}$ www.telessaude.org.br
} 
O estudo de caso foi baseado em uma abordagem qualitativa e quantitativa onde teve caráter exploratório e também estatístico. Como o processo foi misto, a natureza das variáveis foi quantitativa e qualitativa. A variável qualitativa usada para capturar a percepção de alunos no estudo foi a interpretação sobre o objeto estudado, isto é, a compreensão de como os usuários percebem o objeto estudado e quais foram suas experiências sobre o assunto, entender suas ações, seu ambiente e o contexto em que o objeto é inserido. As variáveis quantitativas utilizadas foram coletadas através do questionário System Usability Scale (SUS) para analisar as opiniões, reações, atitudes e hábitos que foram medidos pelos usuários.

\subsection{Participantes}

A população foi composta por alunos de graduação de cursos envolvendo saúde e tecnologia que cursaram a disciplina de informática aplicada à saúde de uma faculdade da Região metropolitana de Recife e que concordaram em participar. Para a realização do estudo foram convidados 259 alunos do $2^{\circ}$ semestre de 2015. Com o objetivo de minimizar o viés, a informação contida nos termos de participação explicou que a contribuição com o experimento seria voluntária e que a informação relatada era confidencial e somente armazenada pelos pesquisadores. $\mathrm{O}$ anonimato de todos os participantes foi garantido.

\subsection{Cenário}

Os participantes foram alunos que frequentaram a disciplina da tecnologia da informação aplicada à saúde. Ao longo do semestre, além do professor inserir todo material didático das aulas da disciplina no INDU, ele fez fóruns de discussões sobre os assuntos lecionados com o objetivo fomentar o conhecimento dos alunos através da troca de informações e também fez perguntas para interagir com os alunos. No final do curso, os alunos foram convidados a responder um questionário de aprendizagem e usabilidade do uso do INDU como ferramenta de aprendizagem do curso de Saúde. Após a coleta de dados, foi realizada a análise de dados com base em abordagens quantitativas e qualitativas.

\subsection{Avaliação de Usabilidade}

De acordo com [ISO 1994], usabilidade pode ser definida com a qual um produto pode ser utilizado por usuários específicos, para atingir objetivos específicos com efetividade, eficiência e satisfação, em um contexto de uso especifico. Surgindo a partir do conceito de interação entre o usuário e maquina, [Nielsen 1993] destaca atributos da usabilidade que servem como parâmetros para a sua efetividade. São eles: capacidade de aprendizagem, eficiência, capacidade de memorização, pouca quantidade de erros e satisfação. Sendo assim, esta sessão tem como objetivo abordar o INDU numa perspectiva de avaliação de usabilidade.

Existem diversas maneiras de se avaliar a usabilidade: testes formais, inspeção, grupos focados, questionários etc. [Jeng 2005]. Com o objetivo de avaliar a usabilidade do INDU, foi utilizada a aplicação de um questionário simples e de rápida aplicação, baseado no questionário SUS (System Usability Scale). Utilizou-se a escala Likert para medir a opinião dos usuários em relação a aspectos como interação, efetividade, frequência de uso, inconsistência do sistema, aprendizagem, dentre outros aspectos destacados por [Brooke 1996] como importantes para a avaliação de um sistema do 
V Congresso Brasileiro de Informática na Educação (CBIE 2016)

Anais do XXVII Simpósio Brasileiro de Informática na Educação (SBIE 2016)

ponto de vista de usabilidade. Esta escala pode ser definida da seguinte maneira: 1 (discordo plenamente), 2 (discordo), 3 (neutro), 4 (concordo) e 5 (concordo plenamente). Também foi adicionado ao questionário um campo de texto, no qual o usuário poderia colocar qualquer comentário sobre o sistema, de forma a disponibilizar um espaço para sugestões, criticas e também elogios. Os questionários foram criados e enviados para os alunos através da ferramenta de formulário do Google Documento, os dados coletados foram armazenados em ferramenta planilha do Google Documento e na análise descritiva estudou-se a média dos resultados das respostas.

As questões do questionário foram divididas nessa ordem, tratando dos aspectos:

- Frequência de uso

- Complexidade

- Facilidade ao usar o sistema

- Auxilio para usar o INDU

- Funções integradas

- Inconsistência

- Aprendizagem rápida

- Sistema é complicado

- Confiança para usar o sistema

- Necessidade de conhecimento prévio para utilizar o sistema

Para o calculo da pontuação, as questões impares (1, 3, 5, 7 e 9) tiveram a sua pontuação subtraída de um e, para as demais questões, subtraiu-se de 5 a pontuação obtida em cada uma. Após isso, cada somatório foi multiplicado por 2.5 para que se atingisse o percentual entre 0 e 100 predefinido no cálculo da SUS, como descrito em [Sauro 2011]. O valor de cada questão é levado em consideração para o calculo do SUS mediante a resposta de cada usuário especifico e, ao final, a media dessa pontuação resulta na pontuação SUS geral que o sistema obteve a partir da aplicação deste questionário entre todos os 103 usuários.

\section{Resultados}

\subsection{Perfil Demográfico e Tecnológico}

O questionário de perfil demográfico foi respondido por 157 alunos (60,61\%). De acordo com a análise dos resultados do perfil demográfico e tecnológico dos participantes, a idade média dos alunos era de 26 anos. Cerca de 38,9\% dos participantes tem a renda média de 2 salários mínimos brasileiros. Além disso, a maioria deles tem mais de um equipamento tecnológico. $\mathrm{O}$ equipamento mais frequente entre os usuários é a televisão (97,5\%) e o menos frequente é o Tablet (29,3\%). (Gráfico 01).

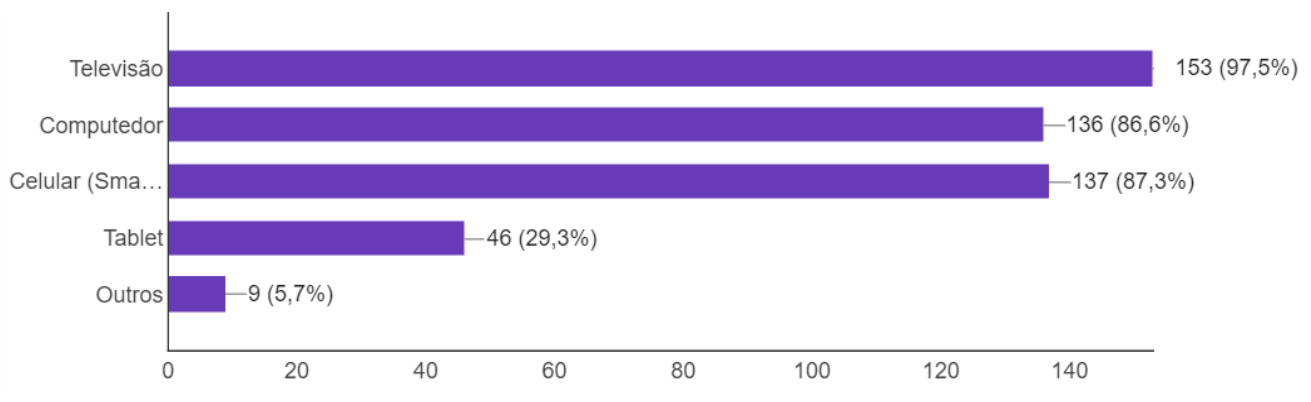

Gráfico 1 - Lista de Equipamentos Tecnológicos 
De acordo com o gráfico 01, o computador e o celular são equipamentos que a maioria dos participantes possuía. Este resultado mostra que o uso de dispositivos móveis não é comum entre os participantes por algumas razões, incluindo o alto preço do móvel equipamento, baixa renda familiar e a cultura da região em não utilizar equipamentos tecnológicos entre profissionais de enfermagem.

Também pode ser notado no resultado da pesquisa que a média de computadores por estudante é maior do que 1 computador. Por outro lado, podemos analisar nos resultados de questionários que a maioria dos alunos $(94,3 \%)$ tinha acesso à internet e que 70,7\% tinham acesso à internet por conexão 3G ou 4G.

Entre os muitos lugares onde os alunos acessam a internet, a casa foi o lugar que teve a maior pontuação média $(94,9 \%)$. Esta informação demonstra que todos os usuários tinham acesso de alguma forma ao INDU. Outro ponto relevante que observamos no estudo foi que os estudantes acessaram a Internet por vários propósitos, entre os propósitos mais citados podemos destacar: estudo $(95,5 \%)$, pesquisa $(87,9 \%)$ e acesso a e-mail $(84,7 \%)$. Além disso, a frequência média de tempo de acesso a Web para $74,40 \%$ dos alunos foi de 07 dias.

\subsection{Avaliação de Usabilidade}

O questionário foi respondido por 103 dos 157 alunos $(65,60 \%)$ que participaram do questionário de perfil demográfico e tecnológico do experimento e cursaram a disciplina de Informática aplicada a Saúde, tendo utilizado o INDU como objeto de aprendizagem durante o semestre. Destes, 100 alunos (o que representa mais de 97\% do quorum total da avaliação), utilizaram o sistema constantemente durante o semestre.

Pela análise dos resultados do questionário de usabilidade SUS, o INDU alcançou a pontuação de 70.99, e de acordo com [Bangor, Kortum e Miller 2009], por essa pontuação, pode-se considerar que o sistema é BOM em termos de usabilidade.

Com o objetivo de analisar separadamente cada aspecto destacado em [Brooke, 1996] e tomando como base a metodologia utilizada em [Simoes e Moraes 2010], onde valor das respostas da escala likert de cada questão foi contabilizado individualmente, foi realizado um somatório da pontuação de cada uma das dez questões de cada aluno. $\mathrm{O}$ gráfico 2 mostra a quantidade total de pontos que cada questão obteve somando-se as respostas dos 103 alunos que participaram da avaliação. 


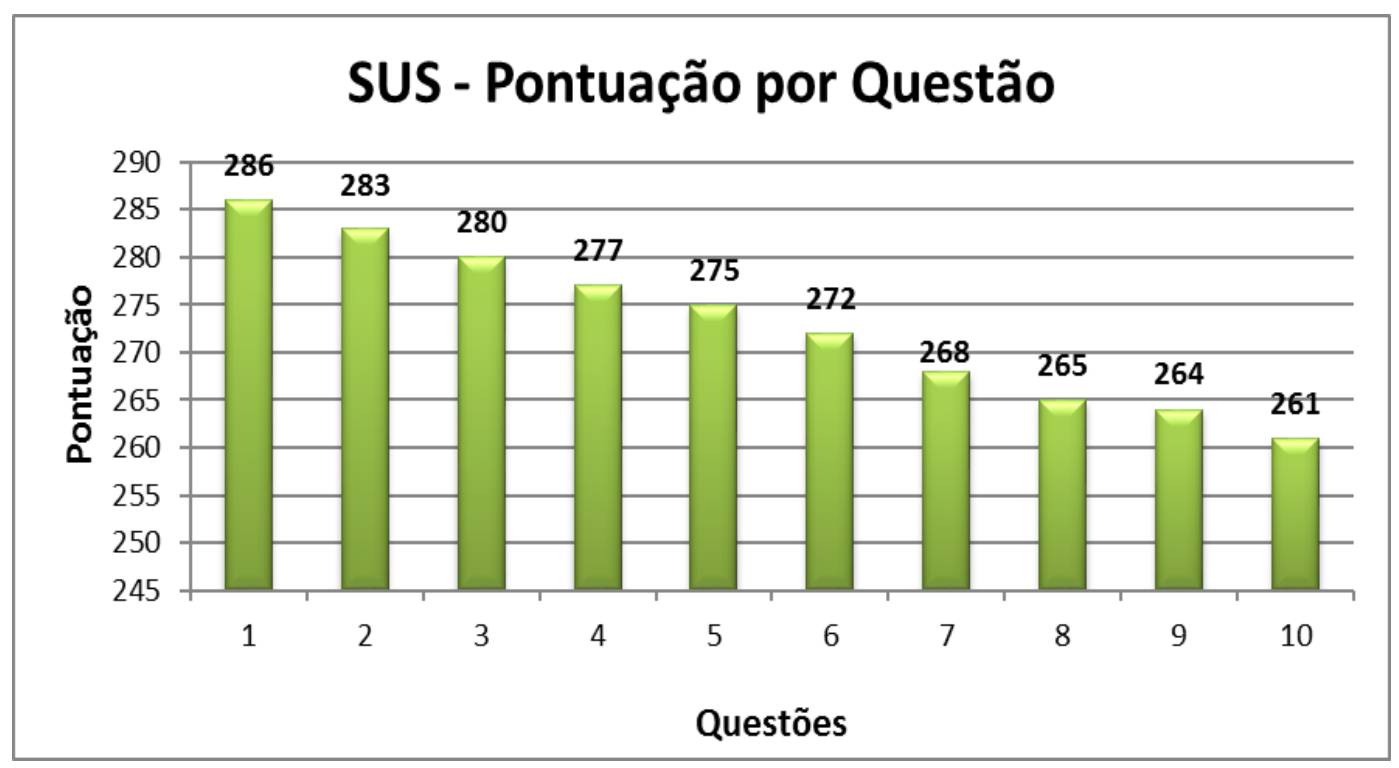

Gráfico 2 - Resultado do questionário SUS por questão

O fato do INDU centralizar diversos artefatos utilizados durante o processo de aprendizagem, como atividades, materiais de estudo, resultados e etc., faz com que o aspecto que expressa a frequência de uso do sistema seja o mais bem avaliado. A segunda maior pontuação que reflete a não-complexidade do sistema do ponto de vista do usuário deve-se à linguagem fácil e direta utilizada para dispor cada elemento e também à organização destes elementos em cada curso especifico, facilitando o entendimento.

Apesar da possibilidade de entenderem rapidamente o que estava presente no sistema, alguns problemas reportados durante o processo de recuperação de senha e autenticação no sistema fizeram com que a questão que avalia a confiança ao usar o sistema tivesse a segunda pior pontuação no questionário. Por fim, a falta de afinidade dos alunos com a plataforma MOODLE, na qual o INDU foi desenvolvido, fez com que os alunos sentissem a necessidade de entender como funciona esta plataforma para utilizarem melhor os recursos ofertados e isso causou a mais baixa pontuação no quesito de necessidade de conhecimento prévio para utilizar o sistema, avaliado na questão 10.

Na questão aberta, que teve oitenta e um usuários respondentes, houveram três assuntos mais recorrentes (pelo menos cinco ocorrências):

- Layout - Os usuários acreditam que o Layout poderia ser melhorado, de forma a dispor um menu mais visível e informações importantes (como nome do curso e/ou atividade) de maneira mais fácil.

- Cadastro - a quantidade de informações necessárias para o cadastro foi questionada e também a complexidade da senha (exigência de caracteres especiais, números e letras maiúsculas e minúsculas).

- Redefinição de senha - Alguns usuários precisaram redefinir a senha e tiveram dificuldades nesse processo.

Por sua própria proposta, o INDU como objeto de aprendizagem, engloba diversas ferramentas e funcionalidades que, do ponto de vista do usuário, são utilizadas separadamente, concentrando tudo em um só lugar. Alunos de computação ( $49 \%$ dos usuários que responderam o questionário) que cursaram a disciplina tiveram mais 
V Congresso Brasileiro de Informática na Educação (CBIE 2016)

Anais do XXVII Simpósio Brasileiro de Informática na Educação (SBIE 2016)

facilidade em utilizar o sistema, uma vez que todos responderam "concordo" ou "concordo plenamente" para a pergunta sobre facilidade de utilização.

Uma questão importante levantada por alguns alunos ainda no espaço para comentários é a possibilidade de usar o INDU em dispositivos mobile: uma vez que se trata de uma tendência mundial, trazer este ambiente para esta abordagem poderá potencializar sua utilização e, consequentemente, efetividade em cumprir com os objetivos aos quais se propõe.

\section{Conclusão}

Nós apresentamos um Ambiente de Inovação Educacional (INDU) como apoio do ensino-aprendizagem na disciplina de tecnologia da informação aplicada a saúde de curso de graduação de enfermagem e nutrição. INDU foi usado como uma ferramenta interativa para ver todos os conteúdos da disciplina, integrando os princípios da aprendizagem.

Com os resultados, observamos que o uso de tecnologia da informação e comunicação não era um hábito comum entre os alunos de um curso de graduação do curso de enfermagem e nutrição, mas eles forneceram benefícios significativos para aprender, para melhorar o processo de ensino, facilitando a troca de informações, onde o objetivo não é apenas para compartilhar, mas também construir uma forma de interação entre alunos.

Apesar de apenas uma parte dos participantes do experimento usou o INDU como uma ferramenta de apoio à aprendizagem, os resultados deste estudo corroboram a análise de usabilidade e aprendizagem. No entanto, há evidências de que a preferência dos usuários na ordem de $95,5 \%$ em favor do uso INDU como ferramenta de apoio ensino e relacioná-la com a sua simplicidade como evidenciado pelas palavras de alguns participantes na seção de análise de satisfação. Em relação variável usabilidade, os alunos, apesar de não ter o perfil tecnológico, gostava de usar o INDU como ferramenta de suporte para a aprendizagem e se sentiam mais satisfeitos e atualizados com o conteúdo da disciplina.

As contribuições previstas neste estudo estavam relacionadas com a análise da usabilidade do INDU e na melhoria na aprendizagem devido à sua utilização, uma vez que era possível realizar as análises em cenários reais no contexto da disciplina de informática no curso de enfermagem e nutrição, criando uma oportunidade para a discussão e reflexão sobre o uso de redes sociais como ferramenta de apoio no processo de ensino e aprendizagem.

\section{Referências}

Bangor, A.; Kortum, P.; Miller, J. (2009). Determining What Individual SUS Scores Mean: Adding an Adjective Rating Scale, J. Usability Studies 4, 3, 114-123.

Brooke, J. (1996) SUS: A Quick and Dirty Usability Scale.

Campos Filho, A. S.; Novaes, m. A.; Gomes, A. S. (2015). A 3D visualization framework to social network monitoring and analysis. Comput. Hum. Behav. 49, C (August 2015), 623-634. DOI=http://dx.doi.org/10.1016/j.chb.2015.03.053. 
V Congresso Brasileiro de Informática na Educação (CBIE 2016)

Anais do XXVII Simpósio Brasileiro de Informática na Educação (SBIE 2016)

Curran, V.R.; Fleet, L.; Kirby, F. (2006). Factors influencing rural health care professionals' access to continuing professional education. Aust. J. Rural Health, v.14, n.2, p.51-5.

Graeff-Martins, A.S. et al. (2008). Diffusion of efficacious interventions for children and adolescents with mental health problems. JCPP, v.49, n.3, p.335-52.

International Standards Organization (ISO). (1994). Ergonomic requirements for office work with visual display terminals. Part 11: Guidance on usability (ISO DIS 924111). London: International Standards Organization.

Jeng, J. (2005). "What is usability in the context of the digital library and how can it be measured?" Information Technology and Libraries Vol. 24 Iss. 2 Available at: http://works.bepress.com/judy_jeng/7/.

Knowles, L. et al. (2008). Disseminating health disparities education through telelearning. JSCI, v.6, n.4, p.78-82.

Nielsen, J. (1993) Usability engineering. Cambridge, Mass.: Academic Pr.

Novaes, M. A., et al. (2012). Tele-educação para educação continuada das equipes de saúde da família em saúde mental: a experiência de Pernambuco, Brasil. Interface (Botucatu) [Internet].

Sauro, J. (2011). Measuring Usability With The System Usability Scale (SUS). Disponível em: http://www.measuringu.com/sus.php. Acessado em: 07 Junho 2016.

Simoes A. P., Moraes A. (2010). Aplicação do Questionário SUS para Avaliar a Usabilidade e a Satisfação do Software de EAD. $10^{\circ}$ Congresso Internacional de Ergonomia e Usabilidade de Interfaces Humano-Computador. PUC - Rio de Janeiro.

WHO - World Health Organization. (2016) eHealth. Genebra: WHO. Disponível em: $<$ http://www.who.int/topics/ehealth/en>. 2016. Acesso em: 30 Mai. 2016.

Zollo, S.A. et al. (1999). Tele-education in a telemedicine environment implications for rural health care and academic medical centers. J. Med. Syst., v.23, n.2, p.107-22. 